
\title{
25 Research Square \\ Relations between influenza, dengue outbreaks, and subsequent bacterial sepsis in French Guiana: a time series analysis
}

\section{Claire Cropet}

Centre Hospitalier Andree Rosemon

Philippe Abboud

Centre Hospitalier Andree Rosemon

\section{Emilie Mosnier}

Centre Hospitalier Andree Rosemon

\section{Loïc Epelboin}

Centre Hospitalier Andree Rosemon

\section{Félix Djossou}

Centre Hospitalier Andree Rosemon

\section{Ward Schrooten}

Centre Hospitalier Andree Rosemon

\section{Milko Sobesky}

Centre Hospitalier Andree Rosemon

mathieu nacher ( $\nabla$ mathieu.nacher66@gmail.com )

Centre Hospitalier Andree Rosemon https://orcid.org/0000-0001-9397-3204

\section{Research note}

Keywords: Influenza, sepsis, dengue, coinfection, French Guiana

Posted Date: March 9th, 2020

DOI: https://doi.org/10.21203/rs.3.rs-16327/v1

License: (c) (1) This work is licensed under a Creative Commons Attribution 4.0 International License. Read Full License 


\section{Abstract}

Objective : Influenza has been shown to increase the risk for severe bacterial infection, in the tropics the seasonality of influenza epidemics is less marked and this may not be the case. Dengue is often followed by prolonged asthenia and some physicians hypothesized increased susceptibility to infections based on anecdotal observations. Time series of influenza and dengue surveillance were confronted with bacterial sepsis admissions to test the hypotheses. Monthly surveillance data on influenza and dengue and aggregated sepsis data in Cayenne hospital were matched between 24/10/2007 and 27/09/2016. An ARIMA $(1,0,1)$ model was used.

Results : The series of the number of monthly cases of sepsis was positively associated with the monthly number of cases of influenza at time t $(\beta=0.001, p=0.0359)$. Forecasts were imperfectly correlated with sepsis since influenza is not the only risk factor for sepsis. None of the ARIMA models showed a significant link between the dengue series and the sepsis series. There was thus no link between dengue epidemics and sepsis but it was estimated that for every 1000 cases of flu there was one additional case of sepsis. In this tropical setting, influenza was highly seasonal, and improved vaccination coverage could have benefits on sepsis.

\section{Introduction}

Coinfections by different pathogens results in agonistic or antagonistic consequences depending on the agents involved. Epidemiological and experimental evidence converge to show that prior respiratory viruses pave the way for invasive bacterial disease. Prior viral infections may thus enhance adherence, colonization and invasion by bacterial pathogens in many different ways: Physical damage to the respiratory epithelium and function, ciliary clearance of bacteria, and bacterial adherence to injured tissues.[1] Immunopathology also facilitates bacterial disease[2].

Dengue fever is an arthropod borne viral disease which leads to large epidemics that often overwhelm health services. In a small fraction of cases, life-threatening severe forms of dengue occur with bleeding manifestations or capillary leakage. [3] Although acute symptoms often recede within a week, a large number of persons however complain of post dengue exhaustion which may persist for weeks after the acute episode. The pathophysiology of dengue is still incompletely understood but it leads to complex immune phenomena. [4]

In the present study, we hypothesized that dengue may lead to transient immunosuppression which could increase the risk of subsequent bacterial infections. Similarly, although influenza has been shown to increase the risk for severe bacterial infection[5], in the tropics the seasonality of influenza epidemics is less marked and this may not be the case.[6]

Therefore we confronted time series of influenza surveillance, Dengue surveillance and admissions in Cayenne hospital for bacterial sepsis to look for any statistical relationship between these viral epidemics and subsequent bacterial sepsis. 


\section{Methods}

\section{Methods}

\section{Data collection}

The study was retrospective and used influenza and dengue data sets collected between 24/10/2007 and 27/09/2016. The surveillance data was obtained from the Cellule Interrégionale d'Epidémiologie Antilles Guyane (Interregional cell for epidemiology) which is responsible for the monitoring of epidemic diseases and records suspected and confirmed cases on a weekly basis from reference laboratories and a sentinel network of clinicians. This surveillance data is used for the health authorities' response and allows them to declare a state of epidemic and unfold appropriate measures. Aggregated sepsis data in Cayenne hospital were collected from the hospital medical information system (CORA software ${ }^{\circledR}$ ). The database was queried using the sepsis International Classification of Diseases codes A390, A392, A400, A401, A402, A403, A408, A409, A410, A411, A412, A413, A415, A418, A419. These codes were pooled in a single "sepsis" variable.

\section{Data analysis}

The analysis used monthly data for each time series. Time series were plotted to look for macroscopic trends. Multiplicative seasonal Autoregressive Integrated Moving Average (ARIMA) models were used to look for a temporal relation between influenza and dengue epidemics, and the number of cases of sepsis.

\section{Model fitting}

Identification stage for the sepsis series - Based on a descriptive analysis (plots) of the monthly number of sepsis cases and of the series' autocorrelation (ACF) and partial autocorrelation functions (PACF), different ARIMA models $(p, d, q)(p s, d s, q s)$ were tested for the sepsis series.

Estimation and diagnostic checking stage for the sepsis series-Models with significant parameters at the $10 \%$ were selected for the final stage. The Ljung Box Q test was used to check the non-autocorrelation of residuals of the selected models. Normality was examined graphically based on the histogram of residuals and on the normality plot.

Final stage - Lags of 0 and 1 month of the series of monthly values of influenza was subsequently incorporated in the previous selected models in order to identify an eventual impact of influenza on the number of sepsis cases. Potential final models included significant parameters at the $10 \%$ level. The Ljung-Box Q test, the residuals histogram and the normality plot were used to confirm that the residual series of the models were Gaussian white noise. Akaike's Information Criterion (AIC), the principle of parsimony, the control of the model variance and the quality of previsions were the performance criteria used to ultimately decide for the final model. A similar reasoning was used for dengue. 


\section{Results}

Description of the different series

Figure 1 shows apparent, but nonsystematic simultaneous peaks of influenza and sepsis.

Data show that except for year 2008, influenza outbreak occurs every year. The magnitude of the epidemics is highly variable across years: a major peak was observed in year 2009 with up to 7000 cases in September. Years 2012 and 2014 also recorded important epidemics. The outbreaks appeared more moderate for other years. The period of influenza activity is also variable across years; thus, though in most cases an intense activity is observed during the first months of the years, is it difficult to identify a clear seasonality.

Time series of sepsis showed a mean number of 14 admissions in hospital for a sepsis per month, varying from 2 to 37 monthly cases.

Figure 2 highlights the 3 dengue epidemics of 2009, 2010 and 2013, with up to 2600, 1500 and 2800 monthly cases identified respectively. The series description does not reveal any obvious trend for a correlation between dengue outbreaks and a subsequent increase in the number of sepsis cases. ARIMA Modeling of sepsis

The identification stage allowed testing different models among which two were retained following the estimation and diagnostic checking stage: $\operatorname{ARIMA}(2,0,0)$ and $\operatorname{ARIMA}(1,0,1)$. Despite the lack of strong seasonal trend, this hypothesis being plausible, it was nevertheless tested but not verified at the end. The fit of the two selected models was correct with parameters that were all significant below $5 \%$, uncorrelated residuals for which the normal distribution hypothesis revealed correct. These two models were thus used to test influenza and dengue as explanatory variables of sepsis.

Sepsis and influenza

Fig S1 shows the cross-correlations between sepsis and influenza series with significant correlations at lags 0 and 1 month, which was judged to be clinically pertinent given the local mucous membrane alterations caused by influenza.

From each of the two previously detailed models of the sepsis series, two new models were declined: one including influenza at time $t$ as input series and one including influenza at time $t-1$. In all four models influenza was significantly associated with sepsis at the $5 \%$ significance level, confirming a correlation between the influenza time series and the sepsis time series.

The final selected model was an ARIMA $(1,0,1)+$ influenza(t). Estimates of the model are shown in supplementary table 1 . 
The series of the number of monthly cases of sepsis was positively associated with the monthly number of cases of influenza at time t $(\beta=0.001, p=0.0359)$. The $\beta$ coefficient of the influenza series thus translated in an additional case of sepsis out of every 1000 cases of influenza.

Fig S1 shows the observed number of sepsis cases in regards to the predicted number of sepsis cases according to the ARIMA model with influenza. Unsurprisingly forecasts were imperfectly

correlated with sepsis since influenza is not the only risk factor for sepsis (Fig S2).

Sepsis and dengue

We integrated the dengue series to the four initial sepsis models testing lags 0,1 and 3 on the basis of a biological relevance. None of the models showed a significant link between the dengue series and the sepsis series. The cross-correlations between dengue and sepsis time series are shown in Fig S3.

The only significant correlations appeared for very long lags that did not seem biologically plausible. Thus, both the descriptive, cross-correlation and modeling approaches led to coherent results which did not show a correlation between the two series.

\section{Discussion}

In interpandemic periods, there are an estimated 1 billion cases of flu, 3-5 million cases of severe illness, and 300 000-500 000 deaths, each year, worldwide[7]. A number of studies have shown that a large proportion of influenza pneumonia in fact results from a synergy between the influenza virus and $\mathrm{S}$. pneumoniae[5]' [8]. The influenza virus increases colonization of the respiratory tract by Streptococcus pneumonia which then facilitates its invasion and the development of pneumonia. This may also occur for other bacteria such as $\mathrm{H}$. influenza or Staphylococcus aureus. Influenza A infections can also have a significant influence on the risk of subsequent meningococcal meningitis[9, 10][11, 12] [13] with variable strength for different influenza subtypes.

Here we observed significant temporal relation between influenza epidemics and the number of cases of sepsis hospitalized in the reference hospital of French Guiana. Despite being close to the equator, influenza seasonality was marked, presumably because of the close link with mainland France where influenza epidemics often affect air travelers to French Guiana who then propagate the epidemic locally. Although the population of French Guiana is small, the output ARIMA model estimates approximately one additional case of sepsis out of every 1000 cases of influenza which during an epidemic is not negligible. Therefore vaccination against seasonal influenza could have non negligible benefits in reducing the number of cases of sepsis. The vaccine for the northern hemisphere is effective in French Guiana, however the timing and the shape of the epidemic are different from what is observed in mainland France.[14] On average influenza epidemics in French Guiana last 22 weeks whereas in mainland France they last 9 weeks. The onset of the epidemic usually occurs 4 weeks after the beginning of the epidemic in mainland France. However, vaccine reimbursement policy only extends until December 31 of a given year. This is likely to limit the number of persons vaccinated and given the 1 sepsis per 1000 influenza cases observed here, extending reimbursement of the vaccine after December may allow benefits on sepsis.[14] 
For Dengue, the initial perception from the bedside was that some patients developed other infections after dengue. This hypothesis was not vindicated by the available data. Therefore, post dengue "immunosuppression" does not seem to have any consequences on subsequent sepsis. Post dengue immune suppression affecting lymphocytes rather than neutrophils, the susceptibility to bacterial sepsis may not have been affected by a prior dengue episode.

\section{Limitations}

The limitations of the present study were possibly the insufficient time span and low patient numbers given the small population of French Guiana which could have masked less obvious interactions. In addition, the sepsis definition was broad, notably because of the small patient numbers when looking at specific diagnoses. The influenza and dengue cases were clinical suspicions which may not have been always accurate. However, they originated from a sentinel network working in close relation to the Health authorities and French Guiana's Pasteur Institutes National Reference Laboratory for arboviroses and influenza. The predicted positive value of the criteria for defining suspected cases by the sentinel network in a proven epidemic context is high, therefore we are confident that the time series reflected the evolution of the studied epidemics.

In conclusion, contrarily to our hypothesis there was no link between dengue epidemics and sepsis. As observed elsewhere there was a significant link between flu epidemics and sepsis. The flu epidemics were highly seasonal and it was estimated that for every 1000 cases of flu there was one additional case of sepsis. In this tropical setting, influenza was highly seasonal, and improved vaccination coverage could have benefits on sepsis.

\section{Abbreviations}

ARIMA

AutoRegressive Integrated Moving Average

ICD

International Classification of Diseases

\section{Declarations}

\section{- $\quad$ Ethics approval and consent to participate}

Participant consent was not necessary as this study involved the use of a previously-published deidentified database according to the Commission Nationale Informatique et Libertés. However, all patients are informed by posters and brochures in multiple languages (including explanatory cartoons for those who cannot read) that the data from medical records may be aggregated and used for research purposes and scientific publications and that they can refuse that their data be included. The retrospective analysis of monocentric anonymous aggregated data is authorized by the Commission 
Nationale Informatique et Libertés and in the above non-opposition conditions. For the present study the data was declared at the CNIL (CNIL number 2108759).

\section{- $\quad$ Consent to publish}

Participant consent was not necessary as this study involved the use of a previously-published deidentified database according to the Commission Nationale Informatique et Libertés.

Availability of data and material: anonymized data can be made available upon reasonable request.

Competing interests: The authors declare no competing interests.

Funding: none.

Author contributions: MN PA conceived the study; CC analyzed data; MN drafted the manuscript; CC, PA, LE, EM, FD, WS, MS critically revised the manuscript for intellectual content; all authors approved the final manuscript; $\mathrm{MN}$ is the guarantor of the paper.

Acknowledgements: Antoine Enfissi from Pasteur institute and Vanessa Ardillon from the CIRE Antilles Guyane for surveillance data.

\section{References}

1. Hament JM, Kimpen JL, Fleer A, Wolfs TF (1999) Respiratory viral infection predisposing for bacterial disease: a concise review. FEMS Immunol Med Microbiol 26: 189-195.

2. de Vries RD1 MA, Geijtenbeek TB, Duprex WP, de Swart RL. (2012) The pathogenesis of measles. Curr Opin Virol 3: 248-255.

3. Halstead SB (2007) Dengue. Lancet 370: 1644-1652.

4. Pagliari C, Quaresma JA, Fernandes ER, Stegun FW, Brasil RA, et al. (2014) Immunopathogenesis of dengue hemorrhagic fever: contribution to the study of human liver lesions. J Med Virol 86: 11931197.

5. Siegel SJ, Roche AM, Weiser JN (2014) Influenza promotes pneumococcal growth during coinfection by providing host sialylated substrates as a nutrient source. Cell Host Microbe 16: 55-67.

6. Hirve SL, P. Paget, J. Vandemaele, K. Fitzner, J. Zhang, W. (2016) Seasonal influenza vaccine policy, use and effectiveness in the tropics and subtropics - a systematic literature review. Influenza Other Respir Viruses 10: 254-267.

7. Organization WH (2016) Influenza. WHO. 
8. Grijalva CG, Griffın, M.R., Edwards, K.M., Williams, J.V., Gil,A.I. , Verastegui, H., Hartinger, S.M., Vidal, J.E., Klugman,K.P., Lanata, C.F. (2014) The Role of Influenza and Parainfluenza Infections in Nasopharyngeal Pneumococcal Acquisition Among Young Children. Clin Inf Dis 58: 1369-1376.

9. Hubert B, Watier L, Garnerin P, Richardson S (1992) Meningococcal disease and influenza-like syndrome: a new approach to an old question. J Infect Dis 166: 542-545.

10. Cartwright KAV, Jones, D.M., Kaczmarski,E. , Smith,A.J. ,Stuart, J.M. , Palmer,S.R. (1991) Influenza A and meningococcal disease. 338: 554-557.

11. Jensen ES, Lundbye-Christensen S, Samuelsson S, Sorensen HT, Schonheyder HC (2004) A 20-year ecological study of the temporal association between influenza and meningococcal disease. Eur $\mathrm{J}$ Epidemiol 19: 181-187.

12. Jacobs JH, Viboud C, Tchetgen ET, Schwartz J, Steiner C, et al. (2014) The association of meningococcal disease with influenza in the United States, 1989-2009. PLoS One 9: e107486.

13. Rameix-Welti MAZ, M.L., Giorgini, D., Ruckly, C., Marasescu, M., van der Werf, S., Alonso, J.M., Naffakh, N., Taha, M.K.. (2009) Influenza A Virus Neuraminidase Enhances Meningococcal Adhesion to Epithelial Cells through Interaction with Sialic Acid-Containing Meningococcal Capsules. Infect Immun 77: 3588-3595.

14. diseases Ecoe (2015) Epidemiological and virological description of influenza epidemics in French Guiana: reflecting on the vaccination calendar. Health Regional Agency.

\section{Table 1}

Table 1. ARIMA model testing the association between influenza and sepsis.

\begin{tabular}{|lrrrrrrr|}
\hline \multicolumn{7}{|c|}{ Conditional Least Squares Estimation } \\
\hline Parameter & Estimate & $\begin{array}{c}\text { Standard } \\
\text { Error }\end{array}$ & t Value & $\begin{array}{c}\text { Approx } \\
\operatorname{Pr}>|\mathrm{t}|\end{array}$ & Lag & Variable & Shift \\
\hline MU & 12.11536 & 1.44390 & 8.39 & $<.0001$ & 0 & Sepsis & 0 \\
\hline MA1,1 & 0.42668 & 0.18353 & 2.32 & 0.0220 & 1 & Sepsis & 0 \\
\hline AR1,1 & 0.77300 & 0.12604 & 6.13 & $<.0001$ & 1 & Sepsis & 0 \\
\hline NUM1 & 0.0010853 & 0.0005105 & 2.13 & 0.0359 & 0 & Influenza & 0 \\
\hline
\end{tabular}

\section{Figures}




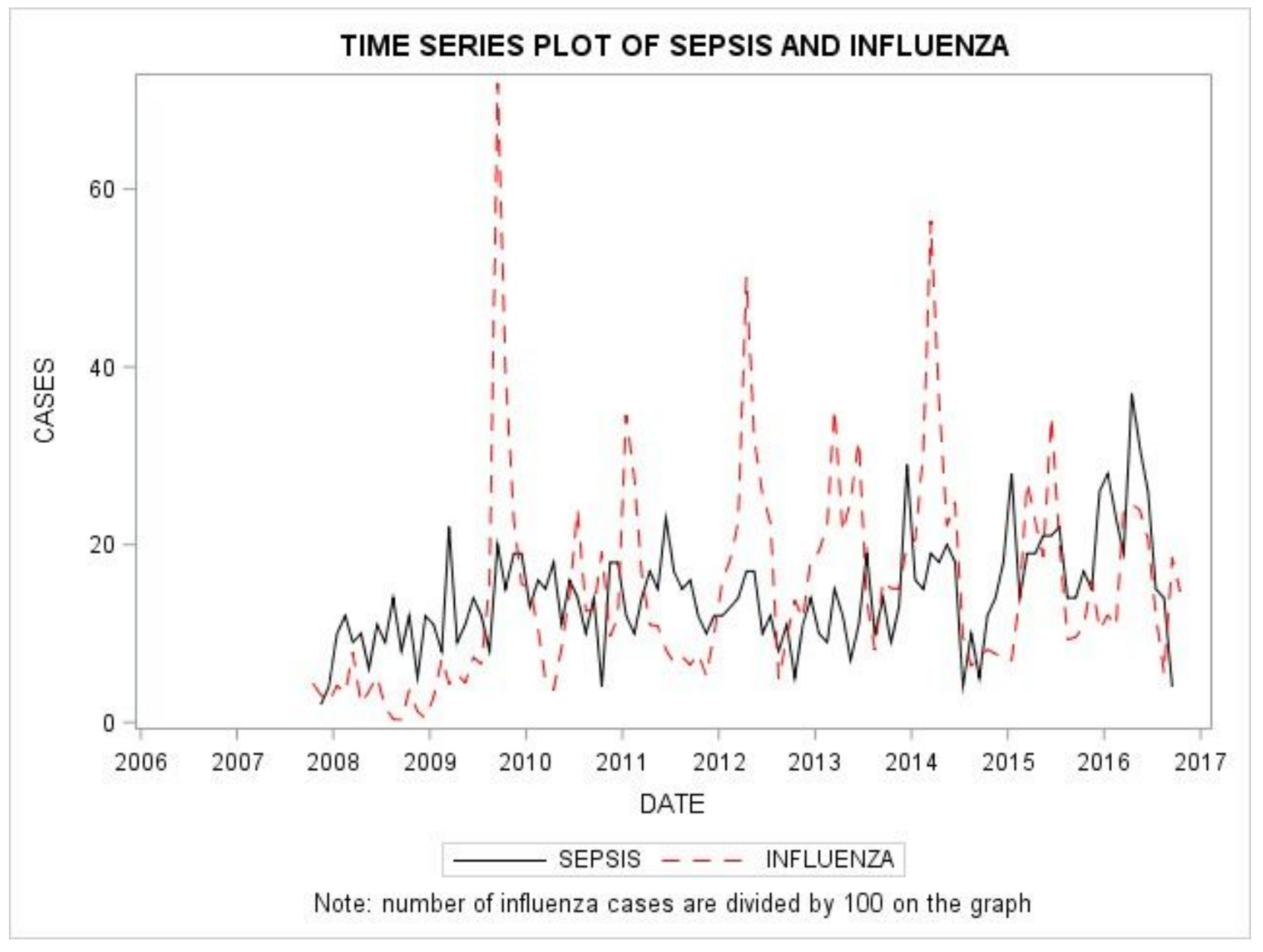

Figure 1

Monthly number of suspected influenza cases and sepsis, French Guiana 2007-2016 


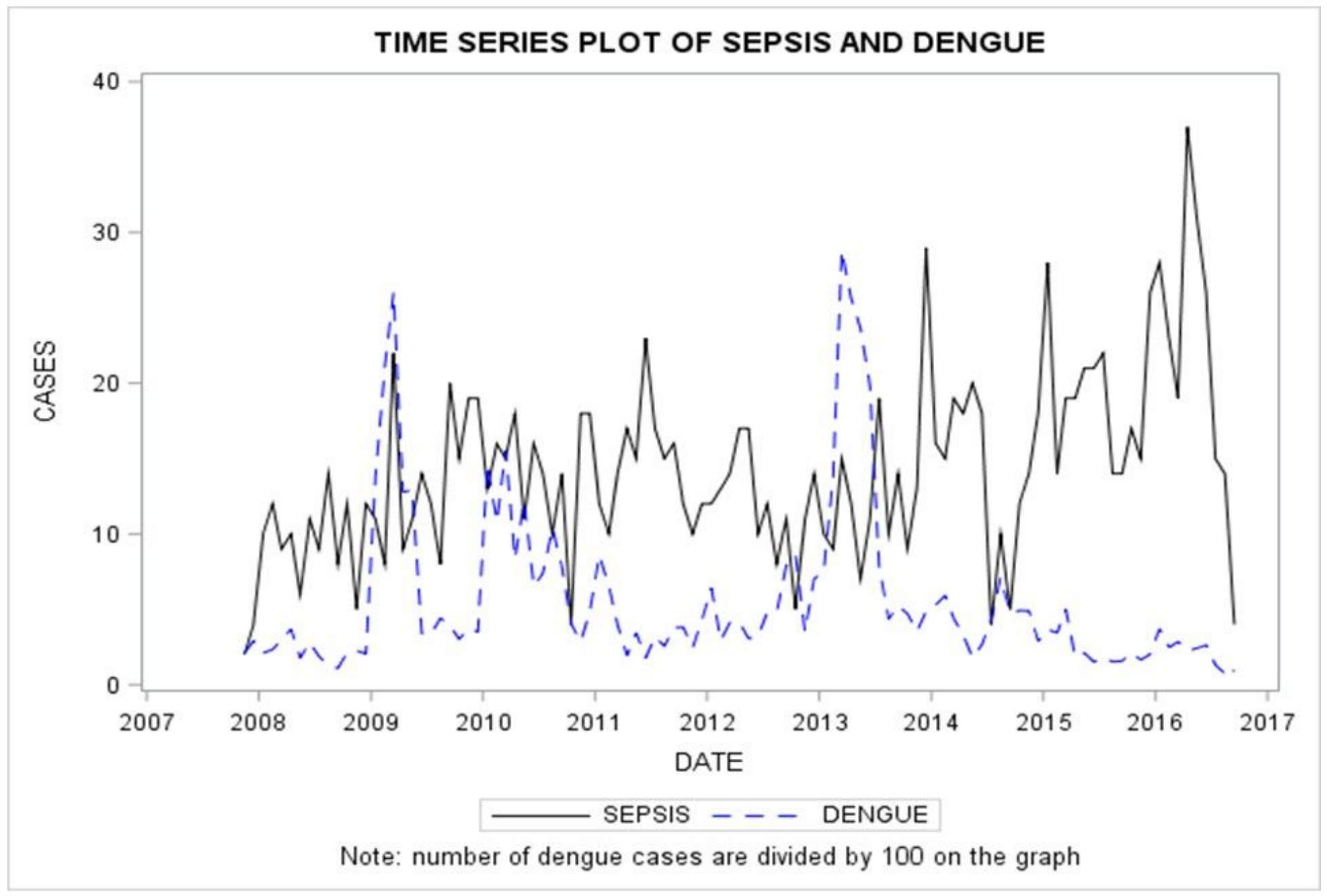

Figure 2

Monthly number of dengue cases and sepsis, French Guiana 2007-2016

\section{Supplementary Files}

This is a list of supplementary files associated with this preprint. Click to download.

- FigS2.docx

- FigS3.docx

- Figs1.docx 